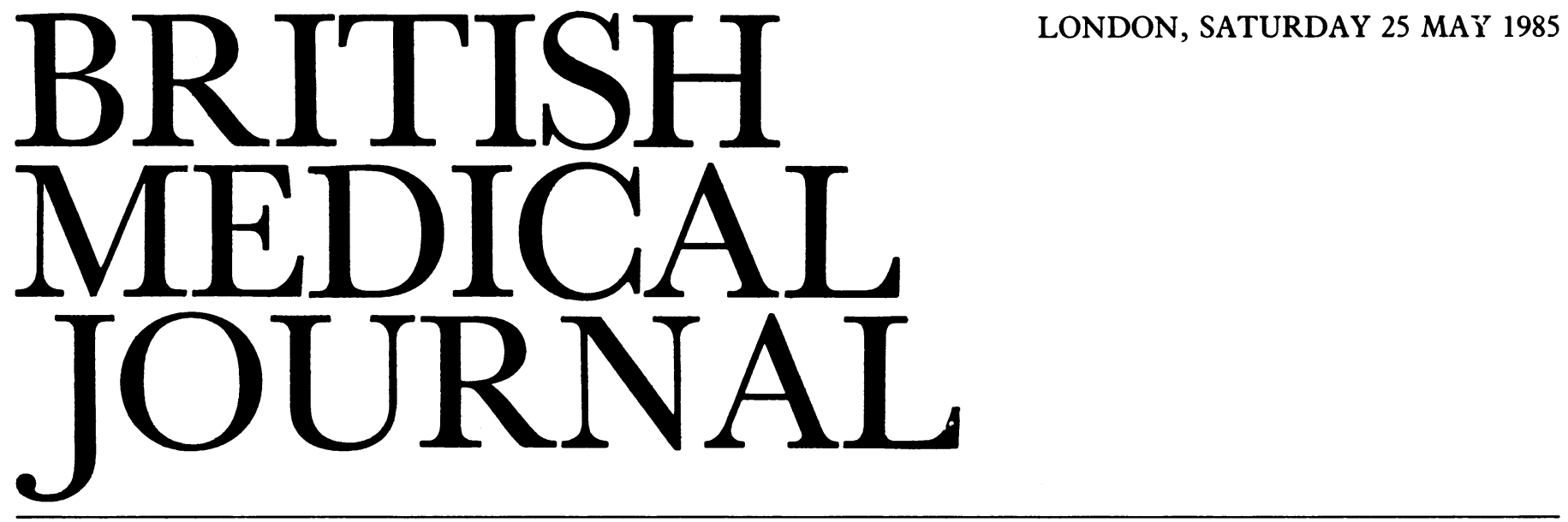

\title{
Resuscitation needed for the curriculum?
}

The paper by Skinner, Camm, and Miles in this issue on the calibre of resuscitation skills found among preregistration house officers at one of our premier teaching hospitals should provide some thought and some activity from the designers of the medical schools' curriculum (p 1549). The investigators found that a mere $55 \%$ of their preregistration house officers could perform the techniques of basic life support: clearance and maintenance of the airway, artificial ventilation by the mouth to mouth method, and external compression of the chest. Theirs is not an isolated finding. Casey found even poorer standards in a comparable group of doctors at Cambridge, ${ }^{1}$ and in 1981 Lowenstein and his colleagues reported that junior residents in a big centre in the United States had similarly ineffective capabilities. ${ }^{2}$

Nor are our young doctors proficient at advanced life support skills, where one might suppose they would come into their own. Skinner et al show that only one in three could pass an endotracheal tube-and that they took an inordinately long time about it. None would have passed the American Heart Association's advanced cardiac life support examination.

Clearly, then, the standards of resuscitation performance among the junior medical staff in Britain leave a lot of room for improvement. Since the general educational standard of our graduates is extremely high in other respects, the fault must surely lie in medical schools not placing sufficient emphasis on this subject.

A survey by questionnaire sent to 30 medical schools in Britain by Smith and Hill from the Southampton Anaesthetic Department produced 18 replies (personal communication). Of these, two thirds offered some training in first aid, but the course was compulsory in only two. All schools claimed to offer training in resuscitation, but the course was compulsory 'in only eight, and the students' knowledge and ability were assessed in only four centres. Most schools did not offer any training at all until the student was well into the clinical years.

As Skinner, Camm, and Miles state, the time is overdue for resuscitation to be given priority in the student curriculum. Within the first few weeks of starting at medical school the undergraduate should receive practical training in emergency aid and basic life support in conjunction with the course in physiology of the cardiopulmonary respiratory system. This training will provide an additional exciting clinical interest at an early stage of training. Spence and Mowbray have shown that first year medical students can learn basic life support techniques in a few hours-and, what is more, they are then capable of acting as competent instructors for members of the lay public such as schoolchildren (personal communication).

The theory and practice of advanced life support should be taught during attachments in the clinical years to departments of anaesthesia, cardiology, or accident and emergency. This skill must also be examined and tested, and passing should be a prerequisite for qualification as a doctorindeed, in certain United States states junior doctors must satisfy the authorities as a condition of employment that they are competent in resuscitation skills.

The Resuscitation Council (UK) has drawn up guidelines for both basic and advanced life support, and these are available on request from the Department of Anaesthetics, Royal Postgraduate Medical School, London W12 1GS.

Training aids are relatively modest in cost, but some thought and expenditure will need to be given to the teachers. Skinner's recommendations that a resuscitation officer should be appointed with the specific task of teaching medical students, nurses, paramedical professions, and doctors (both young and old) is particularly pertinent, and such appointments have already been made in Brighton, Wakefield, and the North East Thames region. If funding is shared between universities and the National Health Service it should not be an overwhelming burden. Resuscitation, provided it is carried out on properly selected cases, represents remarkably good value for money in comparison with many of the other current activities in medicine. ${ }^{3}$

This plea for better training in resuscitation has been made many times before-and many years ago. ${ }^{45}$ Perhaps the difference today is that the mass media are now interested in the matter. It would be better if the General Medical Council and the medical schools put their houses in order voluntarily before they are pressured to do so from outside. Resuscitation is unique in that each and every one of us-at no more than a moment's notice-may be called on to perform it anywhere, both as doctors and as responsible citizens. The 
evidence on our present capabilities shows that most of us will fail when that moment arrives.

Consultant Anaesthetist,

Peter J F Baskett

Frenchay Hospital,

Bristol BS16 1LE

1 Casey WF. Cardiopulmonary resuscitation: a survey among junior hospital doctors. $\mathcal{J} R$ Soc Med 1984;77:921-4.

2 Lowenstein SR, Hansbrough SF, Libby LS, Hill DM, Mountain RD, Scroggin OH. Cardiopulmonary resuscitation by medical and surgical house officers. Lancet 1981 ;ii:679-81.

3 Baskett PJF, Sowden GR, Robins DW'. Fthics in cardiopulmonary resuscitation. American foumal in I:mergency. Hedicine 1984:2:273-4.

+ Brown CG, Sanders AB, Gurley HT, Stair TO, Morkovin V, Jayne HA. Curriculum 101 undergraduate education in emergency medicine. F Med Educ 1984:59:427-9.

5 Anonvmous. Evaluation of medical curricula [Editorial]. Lancet 1972;ii:1014-5.

\section{Infective endocarditis: a preventable disease?}

Sir Thomas Lewis described infective endocarditis as “. . . a progressive disease, ending fatally with so few exceptions that little or no hope of the patient surviving is to be entertained." That was in the preantibiotic era, when the disease was causing about 1000 deaths a year, mostly in patients aged between 20 and $40 .{ }^{23}$ The disease still carries a mortality of about $30 \%$ and causes around 200 deaths a year in England and Wales, ${ }^{+}$but most of the deaths now occur in patients aged 60 or more. 5 The decline in rheumatic fever, the increase in the elderly population, and the appearance of new groups at risk-particularly patients with prosthetic heart valves - have contributed to the changed pattern, while the prognosis has been improved by treatment with antibiotics and valve replacement.

Efforts to prevent the disease have failed-regrettablyas judged by the persisting frequency of streptococcal endocarditis. ${ }^{6}$ The hope that much infective endocarditis should be preventable was based on the findings of workers in the preantibiotic era; they knew that the valves of normal hearts could be infected and destroyed in severe septicaemic illnesses but they recognised a much more common disease, subacute bacterial endocarditis, which appeared to occur only in patients with rheumatic or congenital heart disease and in which a common low grade oral pathogen, Streptococcus viridans, was almost invariably incriminated. ${ }^{.}$Subacute bacterial endocarditis after dental extraction had been reported in $1930,{ }^{7}$ and investigations in the mid-' 30 s showed that dental extractions commonly caused a bacteraemia. ${ }^{89}$ The picture seemed clear: a structural abnormality of the heart was an essential risk factor and dental procedures were the most common cause of the bacteraemia which initiated the illness.

When antibiotics arrived a formula for preventing many cases of infective endocarditis seemed obvious-treat patients with valvular or congenital heart disease before dental procedures. The success of this formula was clearly going to depend on three factors: firstly, that the premises were correct; secondly, that all patients at risk could be identified; and, thirdly, that the selected prophylactic regimens were given correctly and were effective. Unfortunately, difficulties and doubts have emerged on each count. The importance of dental procedures is probably not as great as was originally thought, ${ }^{10-12}$ infective endocarditis often occurs in patients with no known heart disease, ${ }^{13}$ recommended prophylactic regimens have not been followed, ${ }^{14}$ and, finally, no study has ever been performed to show that prophylaxis prevents infective endocarditis.
The current pattern of infective endocarditis and the part played by dental procedures in particular have been put in broad perspective by the results of a study (planned by the British Cardiac Society in association with the Medical Services Study Group of the Royal College of Physicians of London) in which cases of infective endocarditis during 1981 and 1982 were investigated retrospectively by means of a questionnaire. ${ }^{15}$ Unfortunately, reporting was incomplete and, as judged by the mortality statistics from the Office of Population, Censuses, and Surveys and from the Weekly Communicuble Disease Reports, ' ${ }^{\text {in }}$ the cases notified to the investigators were less than one third of the total occurring during the inquiry. Nevertheless, 544 episodes of infective endocarditis were available for analysis making the study the most comprehensive ever undertaken in the British Isles.

The mean age of patients was 52 years and men outnumbered women two to one, pussibly reflecting the higher incidence of bicuspid aortic valves and calcific aortic stenosis in men. Two hundred and thirty patients (43\%) either had normal hearts or a previously unrecognised cardiac abnormality before the onset of endocarditis. Seventy seven (14\%) had prosthetic heart valves. Seventy four patients (14\%) died (mean age 59 years), and there was a differential mortality according to the nature of the infecting organism$30 \%$ in staphylococcal infections, $14 \%$ in infections caused by bowel organisms, and $6 \%$ in other streptococcal infections. The most common infecting organism proved to be $S \mathrm{tr}$ viridans, which was isolated in $262(48 \%)$ of the reported cases. Dental procedures had been performed within the three months before the onset of illness in only 74 patients (14\%), but within this group Str viridans was the infecting organism in $53(72 \%)$. Half of the patients who developed endocarditis after a dental procedure were not in line for prophylaxis because they had no known cardiac abnormality, but in the 37 patients with a known cardiac defect a dental procedure was performed without prophylaxis in 30. Seven patients developed infective endocarditis after a dental procedure despite prophylaxis, and a Str viridans was isolated in four of these. A further 48 patients had not undergone a dental procedure but did have overt dental sepsis, and in 33 $(69 \%)$ of these the infective agent was Str viridans. Thus in $122(22 \%)$ of all reported cases a dental procedure or dental sepsis may have been implicated, but on clinical and bacteriological grounds a non-dental source of infection was considered more likely in 19 reducing the totals to $64(12 \%)$ for dental procedures and $39(7 \%)$ for dental sepsis. Str viridans was the infecting agent in 176 patients in whom no recent dental procedure had been performed and in whom no dental sepsis was apparent. On the grounds that the mouth and nasopharynx are the most likely sources of the most common organism, Str viridans, and that periodontal disease is present in more than one third of our adult population, ${ }^{17}$ poor dental hygiene was thought to represent a greater risk than dental procedures.

In two further papers based on the study Bayliss and his colleagues have provided an overview of the microbiological and pathogenetic features of infective endocarditis in the reported cases. ${ }^{18}{ }^{19}$ Of the 544 episodes, $63 \%$ were caused by streptococci ( $48 \%$ viridans, $15 \%$ other streptococci), $19 \%$ by staphylococci, a wide variety of organisms accounted for a few cases, and $10 \%$ were culture negative. Bowel organisms accounted for $14 \%$ of the episodes. In $60 \%$ of all cases a portal of entry was not apparent; the probable origins of infection in the remainder were dental (19\%), alimentary, genitourinary, respiratory, or skin $(16 \%)$, and invasive procedurés affecting the bloodstream $(5 \%)$. The list of disorders and procedures

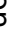

